\title{
Phenomenal Contrast Arguments: What they Achieve
}

\author{
Marta Jorba (University of the Basque Country) \& \\ Agustín Vicente (Ikerbasque/University of the Basque Country)
}

Forthcoming in Mind \& Language

\begin{abstract}
Phenomenal contrast arguments (PCAs) are normally employed as arguments showing that a certain mental feature contributes to (the phenomenal character of) experience, that certain contents are represented in experience and that kinds of sui generis phenomenologies such as cognitive phenomenology exist. In this paper we examine a neglected aspect of such arguments, i.e., the kind of mental episodes involved in them, and argue that this happens to be a crucial feature of the arguments. We use linguistic tools to determine the lexical aspect of verbs and verb phrases - the tests for a/telicity and for duration. We then suggest that all PCAs can show is the presence of a generic achievement-like phenomenology, especially in the cognitive domain, which contrasts with the role that PCAs are given in the literature.
\end{abstract}

\section{Cases of phenomenal contrast argument}

Phenomenal contrast arguments (PCA) are normally employed as arguments for arguing that a certain mental feature contributes to (the phenomenal character of) our experience or is phenomenologically manifest (Siewert 1998; Kriegel 2007). Such arguments play an essential role within the project of determining the admissible contents of experience (Tolliver 2007; Siegel 2010) as well as within the project of vindicating different kinds of sui generis phenomenologies such as cognitive phenomenology (Strawson 2010; Kriegel 2015; Chudnoff 2015a). PCAs paradigmatically present subjects with two scenarios that are alike in most respects except from one (like minimal pairs in Linguistics). Then they ask subjects to imagine being in the first scenario, moving afterwards to the second. Subjects are invited to acknowledge that there is a phenomenal contrast when they move from the first to the second scenario. It is argued that, given that the scenarios differ in only one relevant aspect, the phenomenal contrast should be accounted for by appealing to this specific element, which can thus be said to contribute to the phenomenal character of experiences. This is, in general, the form that PCAs take.

Here we want to put forward an examination of a so far neglected aspect of PCAs, namely, the kind of mental episodes involved in them. We want to explore 
whether the temporal and telicity aspects of mental events is relevant for construing a convincing PCA. We will show that most PCAs in the literature involve punctual and telic mental events, which, following the linguistic literature on lexical aspect, will be here called 'achievements' (see Vendler, 1957 for the original distinction between activities, states, accomplishments, and achievements). After Dowty (1979), achievements are usually defined as near-instantaneous events that are over as soon as they have begun. That is, they are punctual and typically telic, i.e., they have an endpoint (unlike states and activities). Examples of achievements include spot, notice, find, break, or reach. With this characterization in mind, we will argue that what PCAs really reveal is just that there is some kind of general phenomenology associated to achievements, and in particular to mental achievements. We will defend that, while it is disputable whether some cases of PCA related to perception and agency may involve activities, the PCAs that defenders of cognitive phenomenology have put forward all involve only achievements. This conclusion departs from standard interpretations of PCAs, which, as far as we know, have not acknowledged that extant PCAs involve achievements.

In what follows we will present the main cases of PCAs one can find in the literature and will examine the kind of events involved in them. Although we are particularly interested in PCAs in their relation with cognitive phenomenology, PCAs have been used in many areas of philosophical discussion. In the case of the philosophy of perception, one of the most discussed cases is that of recognitional capacities:

"Suppose you have never seen a pine tree before, and are hired to cut down all the pine trees in a grove containing trees of many different sorts. Someone points out to you which trees are pine trees. Some weeks pass, and your disposition to distinguish the pine trees from the others improves. Eventually, you can spot the pine trees immediately. They become visually salient to you. [...] Gaining this recognitional disposition is reflected in a phenomenological difference between the visual experiences you had before and after the recognitional disposition was fully developed" (Siegel 2006: 491).

The form of the argument of recognition capacities is well described by Helton (2016: 6) in the case of viewing a mandolin:

“(1) There is a certain phenomenal difference between the mental state you have when viewing a mandolin in your naïve state and the mental state you have when viewing a mandolin in your expert state. (2) The phenomenal difference 
between your two mental states is due to the fact that in your naïve state, you do not represent the mandolin as a mandolin, whereas in your informed state, you do represent the mandolin as a mandolin. (3) The 'mandolin' representation (if any) in your expert state is a visual experience. (4) In your expert state, you visually perceive the mandolin as a mandolin."

The phenomenal contrast in the case of recognitional capacities aims to show that kind properties (pine trees, mandolins, etc.) — and so high-level properties - are represented in experience. ${ }^{1}$

As mentioned above, PCAs have been often used in the cognitive phenomenology debate. They are the ones that certainly started and motivated the current discussion on the topic. Strawson (2010 [1994]: 5-6) presents a PCA as follows:

"[D]oes the difference between Jacques (a monoglot Frenchman) and Jack (a monoglot Englishman), as they listen to the news in French, really consist in the Frenchman's having a different experience? [...] It is certainly true that Jacques's experience when listening to the news is very different from Jack's. And the difference between the two can be expressed by saying that Jacques, when exposed to the stream of sound, has what one may perfectly well call . . . 'an understanding-experience', while Jack does not”.

The case of the understanding-experience has become one of the paradigmatic examples of PCAs. Siewert (1998) also proposes a variation of this case, where he presents the example of understanding something in two different ways:

$[\mathrm{O}] \mathrm{n}$ some occasions someone utters a sentence, and you momentarily understand it one way [...] -and then are struck by the realization that the speaker meant something else altogether. [...] One can note a difference in the way it seems to understand it, depending on which way one takes the story. And this is so even if one does not picture anything differently, or picture anything at all, as one interprets it differently (Siewert 1998, pp. 278-279).

Still within the cognitive domain, Chudnoff (2015b: 98) has also presented a PCA in the mode of a glossed phenomenal contrast argument, with respect to the episode of

\footnotetext{
${ }^{1}$ Another case of recognitional capacity in which a PCA has been used is in aesthetic perception: "Let F be the distinctive difference between a Vermeer painting and a Van Meegeren; thus, exercising the ability be the distinctive difference between a Vermeer painting and a Van Meegeren; thus, exercising the ability to recognize instances of $\mathrm{F}$ is the exercise of an ability to distinguish Vermeers from Van Meegerens. A contrast experience can be drawn from the collection of experiences that precedes the ability to distinguish Vermeers from Van Meegerens" (Tolliver 2010: 145).
} 
"seeing" that a proposition is true, or what has been called 'intuiting'. The two cases that are contrasted are these:

"Case 1: You entertain the proposition that if $\mathrm{a}<1$, then $2-2 \mathrm{a}>0$ and do not "see" that it is true. In particular you do not "see" how a's being less than 1 makes 2a smaller than 2 and so 2 - 2a greater than 0; Case 2: You entertain the proposition that if $\mathrm{a}<1$, then $2-2 \mathrm{a}>0$ and do "see" that it is true. In particular you do "see" how a's being less than 1 makes 2 a smaller than 2 and so $2-2 \mathrm{a}$ greater than $0 . "$

Within cognition, the case of understanding and intuition or "intellectual seeing" has been explored in hypothetical scenarios by Horgan and Kriegel, respectively. On the one hand, Horgan (2013: 241-244) presents a case where a person initially in Searle's Chinese-Room scenario moves through a series of stages where the apparent level of his proficiency in Chinese is increased step by step without increasing his actual understanding of Chinese (he uses a monitoring device that does the real work). Horgan compares this person's last stage with the case of a person who does understand Chinese:

"Scenario 5 is the one I now want to focus on, harnessing it for use in an explicit argument by phenomenal contrast. There is a clear mental difference between this guy (as I'll now continue to call him) and another guy we might envision (who I'll call 'the other guy'). The other guy is someone who goes through all the same social-environmental situations as this guy and exhibits all the same externally observable behavior, who has ordinary memory, who understands Chinese, and whose mental life is otherwise just like this guy's. Now comes the key question: What explains the mental differences between this guy and the other guy? The only adequate explanation, I submit — and therefore the correct explanation - is the following: this guy lacks Chinese languageunderstanding phenomenology (and also lacks memory-phenomenology), whereas the other guy (who is psychologically normal) undergoes such phenomenology. Hence, by inference to the best explanation, ordinary human experience includes language-understanding phenomenology (and also memory phenomenology)".

On the other hand, Kriegel (2015) puts forward the character Zoe, a sensory-algedonicemotional zombie that happens to be a mathematical genius who "[o]ften (...) struggles 
to find the solution of some problem - she feels stuck, if you will. But sometimes a nice thing happens next: suddenly "the coin drops" and she can see, so to speak, how the solution must go". Zoe is, according to Kriegel, a conceivable creature and our ability to imagine her would create presumption in favour of the thesis that cognitive phenomenology is manifest in Zoe.

Still a third kind of example in this cognitive domain has also been recently presented by García-Carpintero (2016: 265-266) in the context of a discussion on the de se thought. The case can be described as the phenomenal contrast there is between having or not having a de se thought, specifically about realizing that a certain thought is a thought about oneself. The case is illustrated with Perry's (1979: 33) Messy Shopper case:

"I once followed a trail of sugar on a supermarket floor, pushing my cart down the aisle on one side of a tall counter and back the aisle on the other, seeking the shopper with the torn sack to tell him he was making a mess. With each trip around the counter, the trail became thicker. But I seemed unable to catch up. Finally it dawned on me. I was the shopper I was trying to catch.

The phenomenal contrast is thus established between the shopper's thought before the epiphany and after, when he realizes that it is himself who has been doing the mess, and so he acquires a distinctive thought which is about himself (García-Carpintero 2017: 265).

Besides perception and cognition, in the domain of action some authors have also used PCAs in their argumentation. Horgan, Tienson, and Graham (2003: 327-328) propose this kind of argument to show that there is a sense of performing an action versus just having some body part being moved:

"Suppose that you deliberately perform an action-say, holding up your right hand and closing your fingers into a fist. ... [the experience of doing that] is certainly not like this: first experiencing an occurrent wish for your right hand to rise and your fingers to move into clenched position, and then passively experiencing your hand and fingers moving in just that way".

In extant cognitive PCAs, contrasts typically have to do with realizing, grasping, "seeing" or intuiting, and understanding The PCAs that concern perception and action are more difficult to analyse. Let us begin with Horgan et al.'s argument. This case exploits a contrast between parts of your body being moved and your moving such 
parts. However, it is not clear where the phenomenological difference lies: does the process of "holding up your right hand and closing your fingers into a fist" -or any other bodily action- have a distinctive phenomenology as a process, or is such a phenomenology associated with intending, with fulfilling your intentions, with both, or with yet further events, which may all be punctual? The issue here is that it is not clear what the "sense of agency" amounts to. Pacherie (2007), for instance, distinguishes between the experience of intentional causation, the sense of initiation and the sense of control, which in turn can be analysed in different subcomponents. In a view like this, the phenomenological difference between being moved and moving seems to lie in a series of punctual mental events (intending, initiating, etc.) that are present in one case but not in the other.

What is contrasted in the perceptual case is not clear either. Is the contrast in phenomenology associated with recognizing a tree as a pine tree, or with seeing the tree as a pine tree? Surely one can grant that there is a difference between taking a walk in the woods when all trees are just trees and taking a walk in the woods when you are able to recognize pine trees, elms and beeches. What is not clear is whether the difference lies in that in the latter case each time you look at a tree you recognize it as a pine tree (or elm or beech), or whether the difference rests in some enduring activity that your recognitional capacities facilitate. In his description of the case, Helton (2016) claims that there is a difference in "perceiving as", which can be an activity (see below). However, we cannot be sure that the contrast in phenomenology extends beyond the moment when one recognizes the mandolin as a mandolin, or the pine tree as a pine tree. The options available at this point are the following:

(i) The contrast between "living" in a "flat" world and living in a world with mandolins and/or pine trees is that in the latter case each time you bump into a mandolin/pine tree you recognize it as a mandolin/pine tree. This takes place in a moment, and the experience fades away almost immediately. When you take a walk in the woods, each time you spot a pine tree (or the same pine tree in different moments) you experience something related to your recognitional capacities. You may think that your experience is of a process, but actually it is just a series of punctual experiences.

(ii) The contrast between living in a "flat" world and living in a world with mandolins and/or pine trees is that in the latter case each time you bump into a mandolin/pine tree you recognize it as a mandolin/pine tree, and from then on, you see the mandolin/pine tree in a different way, namely as a mandolin/pine tree. The trigger is 
a punctual event: every time you are in front of a mandolin/pine tree, you have to first recognize it as a mandolin/pine tree, but once you have achieved such recognition, the way you experience your activity of looking at the mandolin/pine tree is changed.

As we say, it is not clear which of these two options is the right one. Option (ii) implies that, while you are looking at a certain pine tree that you have recognized as such, you are experiencing something different from what you would have experienced hadn't you recognized it as a pine tree. Seeing the pine tree as a pine tree has a proprietary phenomenology, not reducible to a series of recognition events. It is difficult to know whether this is the case, and it is difficult to know whether Siegel's and Helton's PCAs show that this is the case. Their examples seem to reveal that there is a phenomenological difference between not recognizing and recognizing (trees as pine trees, musical instruments as mandolins). It is not as clear that the PCAs also reveal that the person who is looking at a pine tree for one minute, without recognizing in it a pine tree, and the person who is looking at the same pine tree for a minute, once she has recognized in it a pine tree, are in different phenomenological states during that minute. Intuitions are not clear in this case.

In any event, and although we will also comment on this case below, we will mostly focus our discussion on what PCAs in cognitive cases show. Our suspicion, however, is that all PCAs are related to achievements, that is, that also the agential as well as the perceptual contrast cases establish a contrast between the non-presence and the presence of punctual events that leave a trace in phenomenology. If we are right in our suspicion, then it can be hypothesized that such a trace is a general, common one: that of achieving. However, we will limit our claims to the (clearer) cognitive cases, and hold that such cases seem to only reveal that there is some phenomenology associated with achieving something in cognition.

PCAs of all types have been contested and questioned on different grounds. One first kind of replies to particular cases of PCAs is to argue that the contrast exists but it is not phenomenal, or that the phenomenal contrast can be explained by another relevant parameter than the one submitted. The understanding case has been addressed by invoking shifts in attention, the presence of certain emotional elements or different ways of experiencing phonological elements (Prinz 2011). Another kind of skepticism on PCAs comes from the objections to the method itself, beyond the defenders and detractors of the PCAs results. Koksvik (2015), for example, offers a detailed 
presentation and a critique of PCAs, based on the argument that because our mental lives are rich and fluid, many different features would be able to account for the phenomenal difference and, thus, the conclusion that a certain feature of the experience contributes to its phenomenal character is not warranted. Fürst (2018), on the other hand, goes a step further and offers an explanation for the failure of the method, arguing that both sides of the debate perform the task that PCAs demands in a way that influences their posterior judgments on the introspective results. A third kind of reply is directed at one kind of PCAs, namely, hypothetical PCAs. In order to avoid Koksviklike criticisms, i.e. that many things can actually change from one scenario to the other, the defender of PCAs tries to convince readers that the two different scenarios are indeed minimal pairs by way of fixing all those elements that might vary from scenario to scenario. However, by doing this, the cases turn too hypothetical (as in Kriegel's Zoe's case), with the result that readers become disoriented with respect to the intuitions that the case tries to elicit, given that it is not clear anymore whether there is a phenomenal contrast or not (Koksvik 2015; Chudnoff 2015b).

As advanced, in this paper we will look at PCAs from a new perspective, related to the kind of mental episodes putatively involved in them. Our hypothesis is that the type of the events that figure in PCAs is relevant to their results. First, we will show, using linguistic tests, that extant PCAs concerning cognition all involve events that are linguistically characterized as achievements. Then we will argue that extant PCAs seem to establish only that there is a certain proprietary phenomenology related to mental achieving.

\section{Lexical aspect in PCAs}

\subsection{Linguistic tests}

The aim of this section is to analyse the mental episodes involved in the PCAs presented above. The cases include general mental events such as: realizing, grasping, (intellectually) "seeing" or intuiting, and understanding. In the description of the perceptual cases we find verbs such as 'recognizing', 'spotting', and 'seeing-as'.

Now, works on lexical aspect in linguistics generally distinguish four kinds of aspectual classes: accomplishments, achievements, states and activities (Vendler 1957; Kenny 1963; Filip 2011). A paradigmatic example of an accomplishment is draw a circle or build a house, which is a protracted event that involves an end point and temporal extent. Examples of achievements are find, notice, and reach, which do not 
involve any temporal extent: they are punctual. For states, authors normally present love, know, or believe as examples of episodes that obtain for a period of time, do not involve any change and entail no endpoint. Finally, verbs of activities, such as run or swim, present a temporally extended event that does not need an endpoint to be what it is. Accomplishments and achievements are referred to by telic verbs, which are those that present an action tending toward a goal, whereas states and activities are referred to by atelic verbs, that is, verbs that describe situations that are "realized as soon as they begin" (Garey 1957: 106). The crucial factor distinguishing telicity aspect is the notion of "end" or "limit", not agentivity or intentionality (Garey 1957).

The main tests for telicity usually employed in the literature on lexical aspect are three (Filip 2011). First of all, telic verbs ${ }^{2}$ (i) freely combine with in NP modifiers like in an hour (she drew a circle in a hour, she found the place in 5 minutes), whereas atelic ones combine better with for NP such as for an hour (she has smiled for two minutes, she runs for an hour every day). Secondly, telic verbs are straightforwardly compatible with cardinal count adverbials, as in she draw a circle three times or she found the keys twice, and they are also realized in count-quantified existential constructions: 'she drew a circle three times' entails that there were three circles drawn. In contrast, atelic verbs cannot be combined with cardinal count adverbials unless they shift to telic interpretations (*run three times), and they accept quantifiers such as $a$ lot, as in she runs or swims a lot. Thirdly, telic verbs never sanction the implication from $x$ is $\varphi$-ing into $x$ has $\varphi$-ed: 'she is drawing a circle' does not imply that she has drawn a circle. Atelic verbs, in contrast, normally sanction the implication: from she is running we can conclude that she has run. Within the class of telic verbs, accomplishments and achievements differ in their temporal features: accomplishments are durative while achievements are punctual. This difference entails that verbs of accomplishment can be the complement of verbs that refer to endings (finish, stop) whereas verbs of achievements rarely figure in constructions like $S$ stopped Xing (such as *John stopped finding his keys), unless the verb is interpreted as describing iteration. It also implies that achievement verbs typically do not occur in the progressive ${ }^{3}: *$ John was finding his keys or *Sarah was realizing that Peter had not come sound bad, because find and

\footnotetext{
${ }^{2}$ As we will explain immediately below, these tests do not actually serve to distinguish classes of verbs, but classes of VPs or predicates (verbs + arguments). For ease of exposition, we will follow standard presentations that talk about "verb classes".

${ }^{3}$ There are exceptions, as the so-called "slow motion" reading, as in Sarah is finally noticing that John has shaved off his beard (Rothstein, 2004).
} 
realize do not describe events that have a duration. For that same reason, achievement verbs do not accept modification by manner adverbs such as cautiously or attentively.

In the literature on lexical aspect, there is discussion about how to apply the tests in some cases (see below for some complications), as well as about whether the tests can actually draw distinctions in the verb domain, since lexical aspect seems to be a feature not of verbs alone but of whole verb phrases (VPs) (Dowty, 1979, Verkuyl, 1993). The tests, thus, have to be taken with a grain of salt, applied with care, and seen actually as evaluating whole VPs or predicates instead of verbs. However, even if the tests may well not be definitive, they are still regarded as certainly indicative of whether events denoted by VPs are durative and/or telic or not.

\subsection{Analysis of the cases}

We have suggested that the events involved in cognitive PCAs are all events denoted by achievement VPs. Now we want to see whether our hypothesis holds true. Some of the events that proponents of PCAs mention, both in cognition and in perception, are actually prototypical achievements, listed as exemplars of achievements in the literature (Filip 2011, Rothstein, 2004): realizing (in the cognitive domain) and spotting and noticing (in the perceptual domain). Other cognitive cases involve events that can be linguistically expressed by verbs or VP constructions such as grasp (the view/solution/proposition), understand (a sentence/Chinese), apprehend (a meaning/a proposition), and "intellectually see" (that p is true).

The first example presented was the case of understanding a sentence. With the lexical aspect tests at hand, we first see that it can be complemented with in NP but not with for NP:

(1) Jack understood sentence $\mathrm{S}$ in one second

(2) *Jack understood sentence S for one second

Second, understand a sentence admits of cardinal noun adverbials but not quantifiers:

(3) Jack understood sentence S three times.

(4)*Jack understood sentence S a lot.

On the other hand, understand a sentence does not admit the progressive form:

(5) *Jack is understanding sentence S.

and it cannot be combined with stop/finish and cautiously/attentively:

(6) *Jack stopped/finished understanding sentence S.

(7) *Jack understood sentence S cautiously/attentively. 
Siewert's case also involves understanding, though in this case the contrast is not between not understanding and understanding (a sentence $\mathrm{S}$ ), but between understanding a sentence/utterance in a certain way (with a certain meaning), and understanding it in another way. This difference, however, does not change the punctual and telic nature of the understanding event. Understanding an utterance as expressing meaning $M$ is as much an achievement as understanding an utterance simpliciter.

More controversial is the case presented by Horgan (2013). This case compares not understanding Chinese with understanding Chinese. If we pass the tests to the VP understand Chinese, we get the result that the whole VP seems to be able to denote an activity:

(8) CRG (Chinese-Room-guy) understood Chinese in two months.

(9) CRG understood Chinese for two days.

(10) CRG understood Chinese three times (in his life).

(11) CRG understands Chinese a lot.

(12) $\mathrm{CRG}$ is understanding Chinese $->C R G$ has understood Chinese.

(13) CRG stopped/finished understanding Chinese.

Most, if not all (8-13) seem to be ok, which is puzzling to begin with. However, we can distinguish two meanings of to understand Chinese. One refers to an achievement (or at any rate, to a telic event): to understand Chinese, or any other language, can be something that someone achieves, typically after some effort. There comes a moment when you finally are able to understand Chinese, i.e. you understand when people talk to you in Chinese. This reading of to understand Chinese explains the acceptability of (8) and (10), although (10) requires a bit of imagination.

The other reading of understand Chinese refers to an activity, or so it seems. Understanding Chinese can be something that takes time and that is fully realized as soon as it begins (which explains why (12) is good). This dual nature of the meaning of understand explains why it passes tests both for telicity and for atelicity. Incidentally, these kinds of cases are not exceptional: many VPs have different aspectual readings, which is one of the reasons why linguists recommend using the tests with care.

What interests us here is the prima facie activity reading of understand Chinese, as it is a prima facie problem for the view we want to pursue. Note, to begin with, that 
all putative verbs of achievement can be (apparently) turned into activities if their direct object is a bare plural: find a dog is an achievement, whereas find $\operatorname{dogs}$ can be an activity (Krifka, 1998, Rothstein, 2004). A sentence such as John found dogs for an hour is correct. According to Krifka (1998), the interaction between a telic verb and a homogeneous, not quantized or limited, direct object (a mass noun or a bare plural) gives an atelic VP: the event itself is unbounded, not limited. However, unboundedness (i.e. atelicity) can be a result not of the unbounded nature of the direct object, but of the iteration of events: Michael pushed the shopping carts to customers' cars all day (Ramchand, 2008) is unbounded because the iteration of pushing events is not limited. Thus, it can be said that finding dogs is not an activity per se, but an (unbounded) iteration of achievements: John found a dog, then another one, then a third one, etc., until he completed the hour. As we are not told how many dogs John found, the iteration does not have a stated limit (Ramchand, 2008). In this view, the plurality that the sentence refers to is not a plurality of objects, but of events. Surely, John found dogs can have a reading according to which the object is plural: John was walking in the woods looking for wolves, but he found (a pack of) dogs. However, when the sentence refers to an activity instead of to an achievement, the object is not actually plural: what is plural is the number of finding events. The phrase for an hour simply measures the time when the iteration of telic events took place.

Now, it can be said that understanding Chinese is similar to finding dogs. But Chinese, unlike dogs, is not a plural noun. What is understanding a language, though? Understanding a language $L$ is typically assumed to be the competence of understanding an infinitude of sentences with a particular lexicon and grammar, the lexicon and grammar characteristic of $L$. Thus, understanding $L$ consists in understanding a plurality of sentences. As in the case of finding a plurality of dogs, understanding a plurality of sentences is not an activity, but a plurality of achievements. And note that, at any rate, the alleged phenomenology associated with understanding Chinese would be associated not with the activity, if there were such an activity, but with each of the punctual events of understanding each of the sentences in Chinese that CRG (Chinese-Room-guy) processes.

We also presented the cases of intuiting or intellectually "seeing" that a proposition is true (Chudnoff, 2015b). We take it that "seeing" and intuiting (that p is true) are almost synonymous with grasping, realizing and/or apprehending (that $p$ is true). At least, they seem to be as telic/nontelic and durative/non durative as these other 
VPs are. Now, if these verbs or VPs belong to the same family, it is clear that they are all achievements, given that realizing that $p$ is a prototypical achievement (it is telic, and it is not durative). Kriegel's Zoe's case is also one of grasping, discovering, realizing, or "seeing" - in this case "seeing" the solution to a puzzle. It seems that the same can be said about the example concerning de se thoughts: the difference in phenomenology between thinking "that woman's pants are on fire" and "my pants are on fire" is established at the level of realization (that it is $m e$ ). One can also run the tests on Zoe's "seeing" the solution to the puzzle. In (14-17) it is shown that intellectually "seeing" is telic: it accepts in $N P(14)$ and cardinal complements (16). In accordance with telic punctual verbs, "seeing" doesn't seem to admit the progressive, as in (18) and (19), but note that (18) and (19) may sound good under different readings than the one intended here: (18) is correct if the progressive is understood as "about to", and (19) sounds good, especially with stopped, if the sentence is taken as talking about an ability that Zoe had, namely the ability of displaying episodes of "seeing".

(14) Zoe "saw" the solution to the puzzle in five seconds.

(15) * Zoe "saw" the solution to the puzzle for five seconds.

(16) Zoe "saw" the solution to the puzzle three times.

(17) * Zoe "saw" a lot the solution to the puzzle.

(18) * Zoe was "seeing" the solution to the puzzle.

(19) *Zoe stopped/finished "seeing" the solution to the puzzle.

As said above, we are limiting our case to cognitive PCAs. But it is also interesting to analyse the perceptual case so that the difference between one way of understanding it and the other (mentioned above) is made clear. If the contrast between living in a "flat" world and living in a world with pine trees is that in the latter one is able to spot pine trees and to recognize them as pine trees, we have that the difference lies in achievements:

(20) She recognized the pine tree in a second.

(21) * She recognized the pine tree for a second.

(22) She recognized the pine tree three times.

(23) * She recognized the pine tree a lot.

(24) * She was recognizing the pine tree 
(25) *She stopped/finished recognizing the pine tree ${ }^{4}$.

However, if the contrast extends also to the "seeing as" that follows the recognitional event, we seem to have a case where the PCA reveals that there is a mental activity that has a proprietary phenomenology (as shown by (26), (28), and (30), and indirectly by (31); for an explanation of (27) and (29), see below):

(26) She saw the pine tree as a pine tree for several hours.

(27) She saw the pine tree as a pine tree in five seconds.

(28) She saw the pine tree as a pine tree a lot (compared to how little she saw it as a plain tree). ${ }^{5}$

(29) She saw the pine tree as a pine tree three times.

(30) She is seeing the pine tree as a pine tree -> She has seen the pine tree as a pine tree.

(31) She stopped/finished seeing the pine tree as a pine tree.

Once again, this is a case where a VP passes tests for telicity and for atelicity. But, as before (see the case of understanding Chinese), this is the effect of the VP's having two different readings: in one seeing $X$ as an $X$ is synonymous with recognizing (thus the acceptability of (27), and (29)); in the other, the construction refers to an activity (thus (26), (28) and (30)). As we say, the activity in question is not the dubious activity of iterating episodes of recognition, but the activity one would engage in when one keeps looking at a pine tree after having recognized it as a pine tree. In such a case, and assuming that one is actually seeing the pine tree as a pine tree all the while one looks at it, it may be that one is experiencing something that is different were one seeing the pine tree as just a plain tree. We have expressed some reservation with respect to whether the PCA proves this much. At least, we do not have any clear intuition concerning the case. Recognizing pine trees (as pine trees) and mandolins (as mandolins) seem to have an impact on our phenomenology. Does seeing a mandolin as a mandolin for three minutes have a proprietary phenomenology that lasts those three minutes? The answer, we think, requires investigation that goes beyond our aim here.

\footnotetext{
${ }^{4}$ Like the example before, (25) would sound correct if read as an ability that she had.

${ }^{5}$ If sentences (26) and (28) sound odd, change pine trees for ducks in the rabbit/duck picture.
} 
In any case, leaving the perceptual case aside, and focusing again on the cognitive cases, what the analysis putatively shows is that there is a kind of event that stands out, namely, that of achievements.

\section{Discussion: what PCAs show}

\subsection{Achievement-like phenomenology}

Once we have highlighted the presence of achievements in cognitive PCAs, what are its implications? What should we conclude from this analysis? One first possible reaction is to claim that the fact that cognitive PCAs involve achievements does not show anything relevant about the nature of cognitive PCAs. It could be a mere and irrelevant coincidence. However, we think it is striking that we find only achievements in all the paradigmatic cases of cognitive PCAs (and plausible achievement-readings in the perceptual case), a question that has remained unnoticed in the literature. This fact demands some discussion and explanation. What is the diagnosis of the cases? We will argue that what cognitive PCAs show is in fact that there is a kind of cognitive achievement-like phenomenology, that is, a phenomenology associated with achievements in the cognitive domain. This hypothesis will be developed in this section, together with the discussion of alternative explanations and some possible objections.

Let us begin with what we take to be a natural way to misinterpret the overall situation, related to the kind of contribution to phenomenology that achievements can be thought to make. The comparison between the two scenarios in a PCA is usually done in such a way that readers can notice a phenomenal "click" between the two scenarios. This "click", or change in the course of experience is provided by a sudden realization, recognition, grasp, intuition or understanding - all them achievements. However, this phenomenal contribution of achievements, this "click-phenomenology", is not something we can track in all PCAs, but rather it is just present in the cases of intrapersonal PCAs. Intrapersonal PCAs are those that situate the contrasting scenarios within a subject: Siewert's case of understanding a sentence in two different ways, Chudnoff's example of intuiting by entertaining two different propositions, Kriegel's case of Zoe getting the solution to a mathematical problem, or García-Carpintero's example of a subject's realization that a thought is about oneself. Even Horgan, Tienson and Graham's agentive case is intrapersonal, as well as Siegel's recognitional perceptual cases. But there are two cases that are presented as interpersonal PCA, namely, Strawson's and Horgan's understanding case: what one is meant to compare are 
two situations that involve two different subjects (Jack and Jacques in Strawson's case), and the non-understanding Chinese guy with 'the other guy' in Horgan's case. The description of these cases doesn't involve any intrapersonal phenomenal "click", as the contrast is made between two different subjects: readers are not invited to imagine any kind of "click" or change in themselves. Nevertheless, both cases also involve achievements, as both concern understanding. The phenomenological difference between Jack and Jacques lies in that Jacques achieves things that Jack does not. Thus, when we say that cognitive PCAs involve achievements we are not referring to the issue that in most PCAs we are told to imagine feeling a "click" which corresponds to achieving this or that. Our claim is that extant PCAs, at least in the cognitive domain, work only when the difference between one scenario and the other is that only in the second scenario a subject does something that is punctual and telic.

\subsection{Generic vs. specific achievement-like phenomenology}

There is an interesting question that needs to be addressed: is the achievementlike phenomenology to be understood as generic or specific? This question involves distinguishing various levels of specificity for the achievement-like phenomenology: a general cognitive one, a phenomenology specific to understanding (and likewise intuiting, grasping, realizing, etc), an even more specific associated with understanding sentence $S$, or a phenomenology proper to understanding sentence $S$ as expressing content $C$ (specificity at the level of contents). Proponents of PCAs present their specific cases as illustrating a general case of cognitive experience or phenomenology for conscious thought. Strawson (2010), for instance, uses the understanding example to show that there is linguistic understanding experience (2010: 5) in particular, but the case is brought about as an example of cognitive experience (Strawson 2008: 291ff) .Chudnoff (2015b) and Kriegel (2015) focus on the cases of intuiting and grasping to show that there is cognitive phenomenology. Horgan (2013), in contrast, claims that the resulting phenomenology is Chinese-understanding specific ${ }^{6}$ (but not specific to content $c$ or $d$ ), but also takes the case to illustrate cognitive phenomenology in general. Siewert (1998) presents the case of understanding the same sentence in two different ways, the conclusion being that there is a phenomenology related to understanding specifically content $c$ and not $d$ - but again taking this case as showing that cognitive

\footnotetext{
6 "By the end of the sequence, however, the only credible potential explanation for the guy's inability to understand Chinese will be that he lacks Chinese-understanding phenomenology" (Horgan 2013: 241).
} 
phenomenology is not not reducible to imagery (Siewert 1998: 274). Perceptual cases are all content-involving, given that they concern seeing-as $c$ and seeing-as $d$, and their conclusion concerns the level of contents. We thus observe certain variability regarding the level of specificity claimed to be shown by PCAs.

Our contention is, however, that PCAs only reveal the presence of a generic achievement-like phenomenology. In order to show that this generic achievement-like phenomenology can come in different guises or be more specific, such that we could say that there is a phenomenology specific to understanding, realizing, grasping, recognizing, etc., or to understanding sentence $S$ as expressing content $C$, we would need further PCAs or some other supplementary argument. For instance, an episode of understanding should be contrasted via a PCA with an episode of grasping or realizing to see whether the phenomenology is the same or different. In any case, until a case similar to this one can show that there is a phenomenal contrast between specific kinds of achievements, we lack reason to believe that there is a particular experience associated with the particular achievement of understanding (or intuiting, grasping, or recognizing). ${ }^{7}$

Also, the examined arguments do not show that the experience associated to understanding content $C$ is different from the experience of understanding content $C^{\prime}$, or that seeing an elm as a plain tree is different from recognizing an elm as an elm. Instead, as we said above, the phenomenological difference in these cases seems to be also (generic) achievement-like, as it is about achieving something: in these cases, understanding, or seeing-as, in a second, different, way. We believe this interpretation of extant PCAs implies a twist on some readings of what the method shows with respect to the attitude-content distinction. Some PCAs have been interpreted as being about the content side of mental episodes. In perception, for instance, it is assumed that the "pine tree" PCA shows that pine tree is part of the content of experience, i.e. it is represented in perceptual experience. In the understanding cases, PCAs like Siewert's are normally interpreted as having to do with the contents understood: Jackendoff $(2012 ; 118)$, otherwise a denier of cognitive phenomenology, reads them as showing that "[these different readings] sound the same but feel different, because of the difference in the way you understand them". However, PCAs involving two different understandings of a single sentence do not show that meanings make a phenomenological difference or that

\footnotetext{
${ }^{7}$ There are independent arguments that purport to show this specific phenomenology for different attitudes (see Klausen 2008; Jorba 2016; Peterson 2017)
} 
"different readings sound the same but feel different". Rather, they show that there is some phenomenology related to understanding a sentence in a different way from the one in which one originally understood it. This -understanding a sentence in a new way-is an achievement, and the phenomenology involved in these cases is again an achievement-like phenomenology.

The view considered here, thus, implies that the phenomenal contrast concerns the attitude side of mental episodes, as achievements are associated with the attitude and not with the content. It is the contrast between understanding and not understanding (or understanding in a different way) what leaves a trace in phenomenology, not whether what is understood (grasped, etc.) is $p$ or $q$. The same seems true for the perceptual case, if we limit ourselves to recognition: in recognizing something as a mandolin, the contrast is between seeing something in a certain -uninteresting-way and recognizing that something as exemplifying a certain kind. Here what seems to leave a trace in phenomenology is the recognition event, not its content -at least, this is the way we read the PCA-. According to our understanding, a PCA concerning pine trees shows the same as a PCA concerning elm trees, namely, that recognizing a kind makes a phenomenological difference. If our hypothesis is right, then it departs from a widespread way of interpreting PCAs as being content-involving. Achievements make a contribution from the attitude-side.

Our account in turn implies a revision of the views of proponents of PCAs, who, as far as we know, have not singled out this recurrent achievement-like phenomenology factor in PCAs. However, our view is compatible with the claims that proponents of PCAs want to endorse, namely, with there being cognitive phenomenology, agentive phenomenology, or seeing-as phenomenology. Insofar as there are achievements in the cognitive domain, and such achievements have an impact on phenomenology, we can say that there is cognitive phenomenology-only that just of cognitive achievements (i.e. achievements that are associated with cognitive tasks). What PCAs show in general is an achievement-like phenomenology, but relative to each domain of inquiry, we can say that there is a cognitive-achievement-like phenomenology, an agentive-achievementlike phenomenology (perhaps), and so on-only that such phenomenology is not proprietary. In this way, while the results of our analysis are compatible with there being proprietary cognitive (or rich perceptual) phenomenology, we depart from proponents of PCAs in that it is this kind of method per se that shows such proprietary phenomenology. In contrast with detractors of the method (Koksvik 2015, Fürst 2017, 
for instance) we have argued that the method shows something different from what is usually believed: a general achievement-like phenomenology. We take this is the lesson we can extract from the PCAs found in the literature.

\subsection{Only achievements?}

An intriguing question is why all extant PCAs (or at least those that deal with cognition) contrast achievements (understanding, grasping, etc.). Is it because achievements are easier to contrast? Or is it because one cannot contrast activities (vs. not activities, or other activities) or accomplishments (vs. non-accomplishments, or other accomplishments)? On the face of it, we see no reason why a cognitive PCA should involve only an achievement. In other -non-cognitive- domains, PCAs can work with activities or accomplishments, as (maybe) in the above case of seeing-as. Different activities and accomplishments could enter into PCAs-or so it seems-. There is probably a phenomenal difference between walking and running or between running a mile and running a marathon. But, on the other hand, one could build an inductive argument based on the cases we have analysed and (provisionally) claim that PCAs can only show that achievements leave a phenomenological trace in our cognitive lives. The phenomenon is striking, in any case. Prima facie, one could think that PCAs exploit the notorious nature of achievements, i.e., their being "upheavals of thought" (to borrow Nussbaum's (2001) expression): PCAs put you in a situation where you yourself imagine experiencing one of these upheavals. However, as we have insisted, nothing like this happens when you are told to think about the interpersonal case of Jack and Jacques. Jacques experiences achievements, but they are not any kind of upheaval: as a French speaker, he just understands what any other French speaker tells him. If you, as a reader, want to be in his shoes, you only have to think about your own experience understanding your own language. Each time you understand a sentence in your own language you achieve something. However, the experience goes almost unnoticed. Achievements may leave a trace in phenomenology, but it is not the kind of "eureka" experience that Kriegel's Zoe exemplifies.

To be honest, we do not see the reason why only achievements should work in PCAs. But, on the other hand, we are not able to discard that they are the only kind of events that can elicit the contrast in a cognitive PCA. We cannot see how a PCA concerning the activity of entertaining proposition $p$ could work. In any case, our point, to repeat it again, is that extant PCAs in the cognitive domain only show that there is an 
achievement-like phenomenology that also applies to cognitive achievements. This, we take it, is less than what proponents of PCAs usually attempt to show. So it is somewhat puzzling why many authors have fixated on these cases (and only on these). There are other arguments in the literature that attempt to show that each content has its own phenomenological character (Pitt 2004; XX) or that different attitudes present distinctive phenomenologies (Klausen 2008; Jorba 2016, Peterson 2017). This is not the place to evaluate these other arguments. Here we only want to mention that there is a gap between PCAs and the rest of the arguments in the cognitive phenomenology literature: PCAs concern only cognitive achievements; the other arguments are about proprietary, distinctive and individuative phenomenology and other cognitive attitudes, issues with respect to which, if we are right, PCAs do not illuminate us at all.

Our attitude towards PCAs is thus agnostic. We do not really know whether PCAs have the power to bring non-achievement-like phenomenologies into light. Our claim is restricted to what we currently have. And our diagnosis is that PCAs, until now, have only showed that there is achievement-like phenomenology. That is, if you believe that PCAs are the best arguments we have for cognitive phenomenology, you are not (yet) entitled to believe that there is a distinctive and individuative phenomenology in the cognitive domain. The most you get from PCAs is that there is a phenomenology related to achievements, which is also there in the case of cognitive achievements..

That PCAs only seem to work for achievements puts pressure on one idea that may come to mind when confronted with them. This is the idea that the alleged phenomenological difference between situation A and situation B may be an artefact of the argument. It can be that the very contrast could be playing a determining role in establishing the phenomenal contribution and would be generating the phenomenal effect. From this, it could be concluded that PCAs do not report on an existing phenomenological difference but generate the illusion that there is such a phenomenological difference. This possible problem is in fact a general worry that can be raised against PCAs, given the fact that the method relies on there being a contrast in the first place.

There are two main reasons to resist this possibility. First, we can look at the episodes normally involved in PCAs in isolation, and so out of the context of the contrast, and then see whether they seem to contribute to phenomenology in the same way as in the contrasting case. If they don't, then the suspicion that the contrast is playing a determining role looks more prominent; if they do, then the explanation for 
why they contribute in such a way cannot be found in the contrast itself. If we thus take understanding, grasping, recognizing, intuiting as isolated episodes out of contrast contexts, we see that they still "contrast" with whatever episode or experience precedes them. The examples of perceptual recognition and cognitive realization seem to behave in the same way: when considered in isolation, without explicitly embedding them in a PCA, perceptual recognition and cognitive realization still contribute to phenomenology in the form of experiences of punctual change.

However, another powerful reason to discard the idea that the phenomenal contrast is an artefact of the PCA is precisely that PCAs only seem to work for achievements, or, at any rate, that they work best for achievements. If the phenomenological difference were generated by the contrast, we should expect that non-achievement-like episodes such as activities would also clearly enter into PCAs, as the phenomenal contribution would be due to the contrast itself and not to the kind of episode involved in it. However, take the case of seeing-as as an activity. At various places we have said that our intuitions are far from clear in this case. If one compares how clear the case of recognizing is with how unclear the case of activity-seeing-as seems to be, one should wonder why the contrast works so well in the first case and not as well in the second. If it were all a matter of contrasting, we should get the same intuitions in activities as in achievements. But PCAs involving achievements always give better results (or, alternatively, they are the only ones that give good results).

\section{Final remarks}

Phenomenal contrast arguments are widely used in philosophy of mind as purporting to show different things: that certain features contribute to the phenomenal character of experience, that certain features are represented in the content of experience, and that there exists sui generis kinds of phenomenology such as cognitive phenomenology. Despite the discussion around this kind of argument, no attention has been paid to the ontological profile of the episodes involved in PCAs. Once we look at this issue, using linguistic tests for atelicity and duration, we discover that the kind of episodes involved in PCA are achievements- specially clear is the case in cognition and less so in perception, as discussed at length. This result gives us evidence to put forward the hypothesis that PCAs in fact show the presence of a generic achievement-like phenomenology, clearly in the cognitive domain-and less so in perception, though we have suggested a similar interpretation. This conclusion departs from standard 
interpretations of PCAs, which, as far as we know, have not acknowledged that extant PCAs involve achievements. It also introduces a new element in the dispute between defenders and sceptics of this philosophical method.

\section{Acknowledgments}

\section{References}

Chudnoff, E. (2015a). Cognitive Phenomenology. London: Routledge.

Chudnoff, E. (2015b). "Phenomenal Contrast Arguments for Cognitive Phenomenology", Philosophy and Phenomenological Research XCI (1): 82-104

Dowty, D. (1979) Word Meaning and Montague Grammar. The Semantics of Verbs and Times in Generative Semantics and in Montague's PTQ. Dordrecht: Reidel.

Filip, H. (2011)“Aspectual Class and Aktionsart." Semantics: An International Handbook of Natural Language Meaning, edited by Maienborn, Claudia, von Heusinger K. s and Paul Portner. Berlin and New York: Mouton de Gruyter. Pp. $1186-1217$.

Fürst, M. (2017). "On the Limits of the Method of Phenomenal Contrast", Journal of the American Philosophical Association 168-188. doi: 10.1017/apa.2017.25

García-Carpintero, M. (2017). "The philosophical significance of the De Se", Inquiry: An Interdisciplinary Journal of Philosophy 60 (3):253-276.

Garey, H. B. (1957). "Verbal Aspects in French", Language 33, 91-110.

Helton, G.(2016). "Recent Issues in High-Level Perception". Philosophy Compass 11 (12): 851-862.

Horgan, T. (2013) "Original Intentionality is Phenomenal Intentionality". The Monist, 96: 232-251.

Horgan, T., Tienson, J. and Graham, G. (2003) "The Phenomenology of First-Person Agency". In S. Walter and H. D. Heckmann (eds.), Physicalism and Mental Causation: The Metaphysics of Mind and Action. Imprint Academic (2003), 323-40.

Jackendoff, R. (2012). A User's Guide to Thought and Meaning, New York: Oxford University Press.

Jorba, M. (2016). "Attitudinal Cognitive Phenomenology and the Horizon of Possibilities", in Breyer, T. and Gutland, Ch. (2016). Phenomenology of Thinking. Philosophical Investigations into the Character of Cognitive Experiences. London: Routledge.

Kenny, Anthony 1963. Action, Emotion and Will. London: Routledge and Kegan Paul.

Klausen, S. H. (2008) "The Phenomenology of Propositional Attitudes", Phenomenology and the Cognitive Sciences 7:445-462.

Koksvik, O. (2015). "Phenomenal Contrast: A Critique." American Philosophical Quarterly 52 (4): 321-334.

Kriegel, U. (2007). "The Phenomenologically Manifest." Phenomenology and the Cognitive Sciences 6: 115-136.

Kriegel, U. (2015). The Varieties of Consciousness. Oxford: OUP.

Krifka, M. (1998) The origins of telicity. In S. Rothstein (ed.). Events and Grammar. Dordrecht: Kluwer, 197-235.

Levin, B. and M. Rappaport Hovav, 1995. Unaccusativity: At the Syntax-Lexical Semantics Interface. Cambridge: MIT Press. 
Nussbaum, M. (2001) Upheavals of Thought: The Intelligence of Emotions. Cambridge: Cambridge University Press.

Pacherie E (2007) The sense of control and the sense of agency. Psyche 13: 1-30

Peterson, J. (2017). "How to Defend the Phenomenology of Attitudes", Philosophical Studies. https://doi.org/10.1007/s11098-017-0974-6

Pitt, D. (2004). The Phenomenology of Cognition or What Is It Like To Think That P?, Philosophy and Phenomenological Research, 69: 1-36.

Prinz, J. (2011). The Sensory Basis of Cognitive Phenomenology, in Bayne, T. \& Montague, M. (eds.). (2011). Cognitive Phenomenology. Oxford: Oxford University Press. 174-196.

Ramchand, G. (2008). Verb Meaning and the Lexicon. Cambridge: Cambridge University Press.

Rothstein, S. (2004). Structuring Events. Oxford: Blackwell.

Siegel, S. (2006). Which properties are represented in perception? In T. Gendler and J.Hawthorne (Eds.), Perceptual Experience (481-503). Oxford: Oxford University Press.

Siegel, S. (2010). The Contents of Visual Experience. Oxford: Oxford University Press.

Siewert, Ch. (1998). The Significance of Consciousness. Princeton, NJ: Princeton University Press.

Strawson, G. (2010). Mental Reality (2nd edition). Cambridge, London: MIT Press.

Tolliver, J. T. (2007). "Sensing, Perceiving, and Thinking: On the Method of Phenomenal Contrast.” The Southern Journal of Philosophy. 45:143-151

Vendler, Z. (1957). "Verbs and times". Philosophical Review 66:143-160.

Verkuyl, H. J. (1993). A Theory of Aspectuality: The Interaction between Temporal and Atemporal Structure. Cambridge: Cambridge University Press. 